\title{
Atrophic mandible fracture due to dental implants: Case report
}

\author{
Jun-Hyung Jeon', Jung-Gon Lee', Pil-Young Yun', Young-Kyun Kim ${ }^{1,2}$ \\ ${ }^{1}$ Department of Oral and Maxillofacial Surgery, Section of Dentistry, Seoul National University Bundang Hospital, Seongnam, \\ ${ }^{2}$ Department of Dentistry and Dental Research Institute, School of Dentistry, Seoul National University, Seoul, Korea
}

\begin{abstract}
Implant placement in an edentulous area is a common restorative approach. On the other hand, in atrophic posterior mandible cases, the low bone level and the presence of the inferior alveolar nerve can introduce many limitations, along with complications, such as mandibular fractures, dysesthesia, and osteomyelitis. This case report discusses an elderly patient with a mandibular fracture accompanied by osteomyelitis after multiple standard length implant placements in an atrophic mandible. In such cases, clinicians should be aware of the high risk of mandibular fractures and are recommended to place short implants without compensating with wide implants. Despite the efforts when mandible fractures accompanied by osteomyelitis occurs, it is crucial that timely reduction and active surgical treatment of osteomyelitis along with long-term antibiotic treatment be done to avoid further bone loss. (JOURNAL OF DENTAL IMPLANT RESEARCH 2021;40(3):85-89)
\end{abstract}

Key Words: Dental implants, Osteomyelitis, Mandibular fracture

\section{INTRODUCTION}

Loss of teeth in the elderly may be regarded a natural part of aging, however comes with considerable declination of quality of life. The conventional dentistry has offered dentures for restoring the edentulous jaw, however, patient discomfort due to continuous alterations of the alveolar bone as well as incomplete fitting has been a long task. The introduction of dental implants somewhat relieved the problems that dentistry was facing and has now become a universal treatment in dentistry and have been reported with high success rates. However, postoperative complications such as bleeding and hematoma, nerve damage, and infection, as well as peri-implant diseases and prosthetic complications, continue to occur ${ }^{1)}$. Specifically, a mandible fracture can occur when a long or wide implant is placed in a mandible with severe bone atrophy. If this condition is not treated correctly, it can progress to osteomyelitis or severe jaw defects ${ }^{2}$.
Herein we introduce a case of an 88-year-old elderly patient who experienced fractures after multiple implants were placed in the atrophic mandible at a local dental clinic. Osteomyelitis occurred due to delay in treatment, and several surgeries and long-term hospitalization were performed as follow-up treatments. Based on a literature review of the incidence of mandibular fractures after implant placement, we suggest guidelines and prevention protocols, in addition to diagnostic treatment plans to minimize complications in elderly patients with severe mandibular degeneration. This case study was conducted after receiving approval from the Institutional Review Board of Seoul National University Bundang Hospital (IRB No: B-2108-700-701).

\section{CASE REPORT}

An 88-year-old female patient with no underlying disease experienced left mandibular mobility, swelling of the

Received July 20, 2021, Revised August 22, 2021, Accepted August 31, 2021. (c) Journal of Dental Implant Research.

This is an open access article distributed under the terms of the Creative Commons Attribution Non-Commercial License (http://creativecommons.org/licenses/by-nc/4.0) which permits unrestricted non-commercial use, distribution, and reproduction in any medium, provided the original work is properly cited.

Correspondence to: Young-Kyun Kim, https://orcid.org/0000-0002-7268-3870

Department of Oral and Maxillofacial Surgery, Section of Dentistry, Seoul National University Bundang Hospital, 82 Gumi-ro 173 Beon-gil, Bundang-gu, Seongnam 13620, Korea. Tel: +82-31-787-7541, Fax: +82-31-787-4068, E-mail: kyk0505@snubh.org 

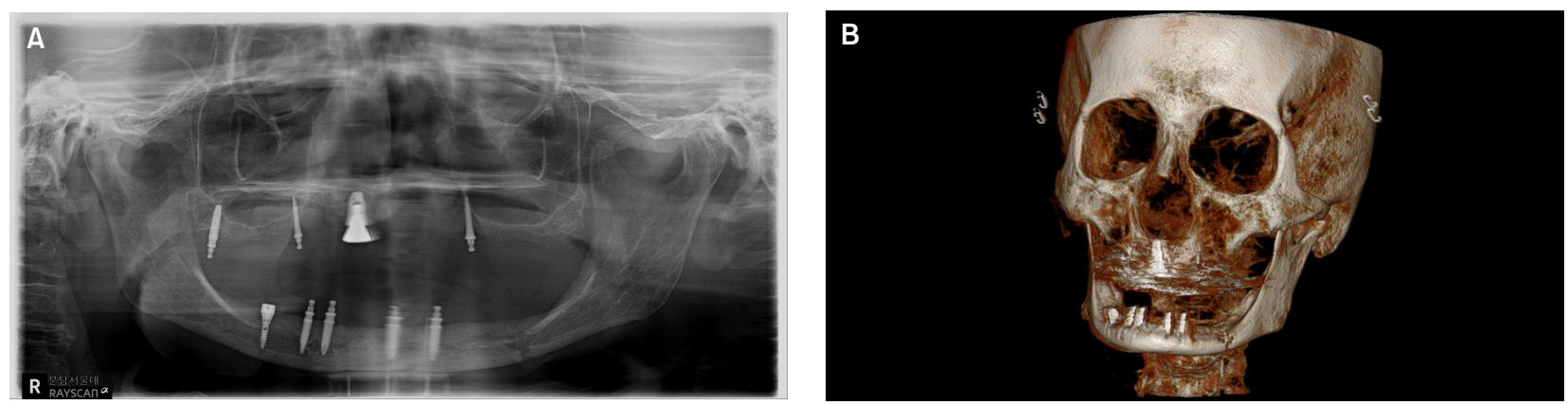

Fig. 1. Preoperative panoramic radiograph (A) and CBCT (B) showing fracture in the left side of mandibular body.

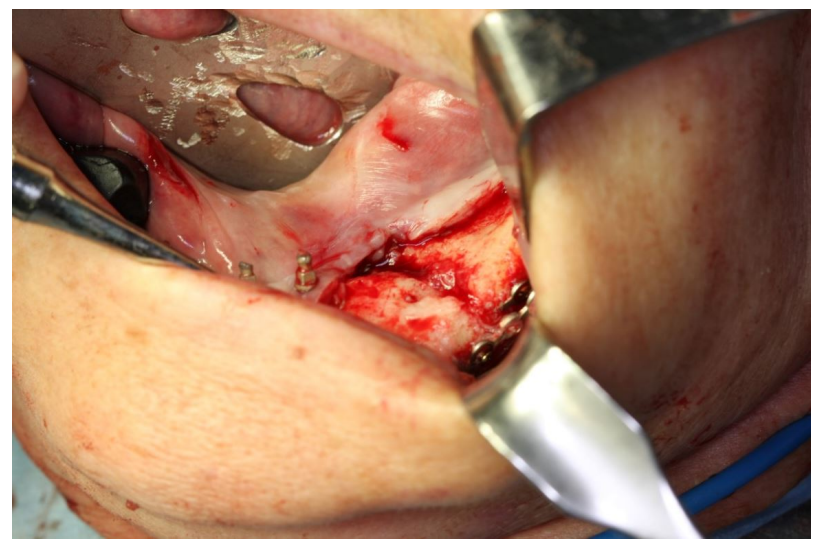

Fig. 2. Fracture site undergoing ORIF and bone graft using Pedistick.

surrounding tissue, and pain after receiving multiple implant placements in the upper and lower jaw for implant-supported overdenture at a local dental clinic. The implant placed in the left posterior part of the mandible was removed, and the patient was referred to our hospital for professional treatment. Clinical and radiological examination revealed radiographic lesions, fracture lines were observed around the removed implant, and osteomyelitis was diagnosed due to a fracture of the mandible (Fig. 1). On February 14, 2018, after the fracture site was exposed under general anesthesia, part of the infectious tissue and collateral bone were removed, and the bone fragments were reduced with two titanium miniplates (Figs. 2, 3). A xenograft (PediStick, Al Mafsal Medical Co., Riyadh) was transplanted into the surrounding bone defect, and wound dressing was performed by administering $1 \mathrm{~g}$ of cefazolin (Chongkundang, Seoul, Republic of Korea) three times a day before the patient was discharged on February 20, 2018. Intermaxillary fixation was not performed because the maxilla and mandible were

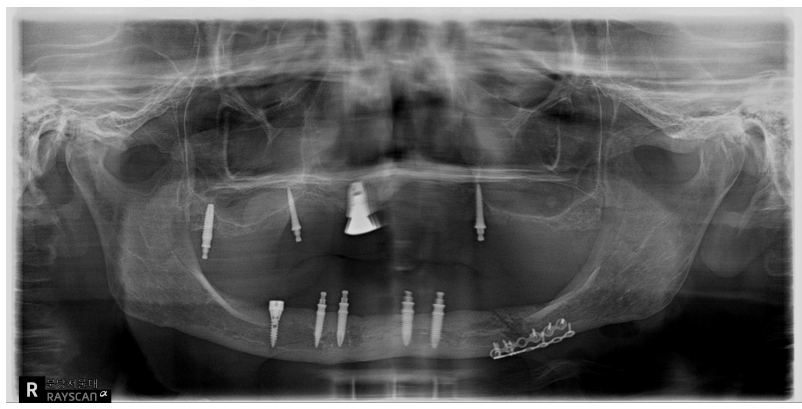

Fig. 3. Postoperative panoramic radiograph of ORIF.

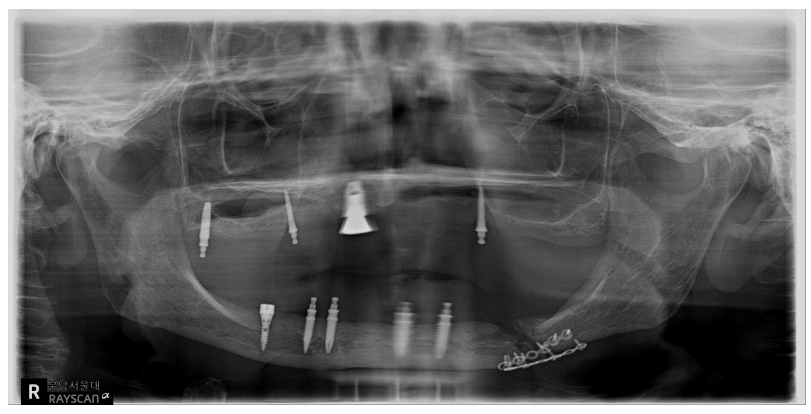

Fig. 4. Panoramic radiograph at one month postoperative showing non-union of fracture with osteomyelitis.

completely edentulous. On April 2, 2018, the patient was re-referred based on swelling, tenderness, fistulas, pain, as well as bone mobility at the local dental clinic follow-up and panoramic radiograph showing non-union of fracture with osteomyelitis (Fig. 4). Wound management was performed with $1 \mathrm{~g}$ cefazolin (Chongkundang, Seoul, Republic of Korea) that was administered three times a day after re-hospitalization, based on a diagnosis of non-union of fracture segments accompanied by osteomyelitis. On April 11, 2018, semi-rigid fixation using a miniplate with an iliac bone graft was performed for reconstruction of a non-union fracture fragment (Fig. 5). 


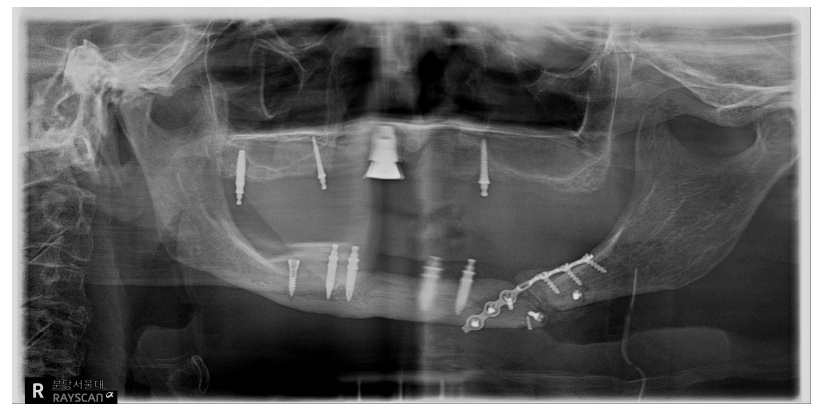

Fig. 5. Postoperative panoramic radiograph after segmental mandible resection and reconstruction with iliac bone graft.

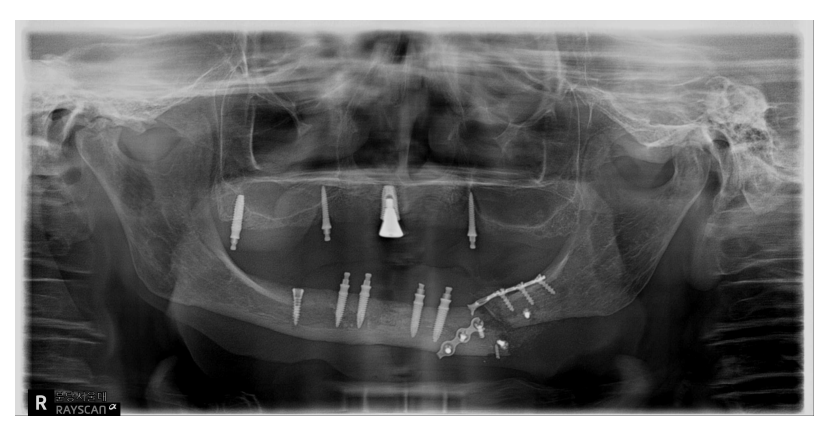

Fig. 6. Panoramic radiograph after re-fracture due to a falling accident in the ward.

On April 24, 2018, severe skeletal mobility with a fractured metal plate was observed due to a fall that occurred during hospitalization (Fig. 6). The operation site showed swelling, local heat, and skin erythema were noted in the lower left mandible; blood test results showed elevated CRP of 4.98 and ESR of 66. During this time, the patient was in a very weak state and was disoriented and expressed delirium. Based on this, we hypothesized that the operation site had re-fractured due to the two falls and conducted IMF using SAS. In addition, a neurologist consultation for intermittent delirium symptoms with poor orientation confirmed that the symptoms were likely caused by risk factors such as advanced age, long-term immobility, post-operative pain, emotional agitation, and infection. The neurologist recommended the patient maintain psychological stability, familiar parental care, and that the patient sleep with a night-light turned on for symptoms that were not severe and that did not required medication. On May 2, 2018, open reduction and internal fixation were performed using a reconstruction metal plate without a bone graft (Fig. 7). Long-term antibiotics were administered, and for the symptoms im-

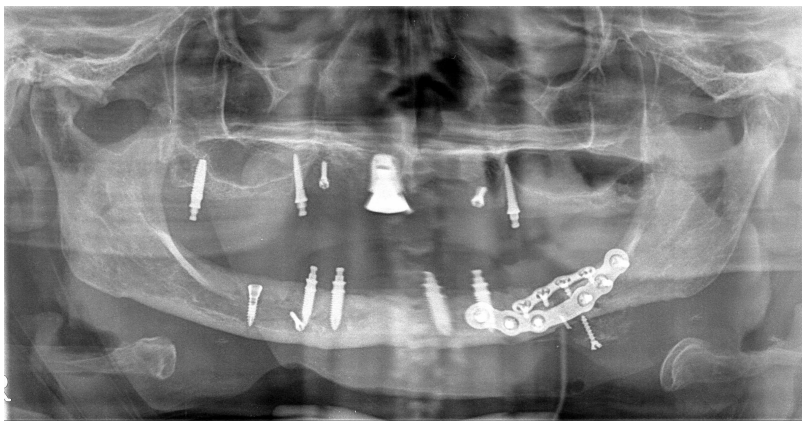

Fig. 7. Panoramic radiograph at one month postoperative after ORIF and insertion of reconstruction plate before the leave of hospital.

proved upon follow-up, the patient was discharged on June 3, 2018. After the discharge, the patient did not come in for follow-up checks.

\section{DISCUSSION}

This case introduces a full edentulous patient with severe mandible atrophy who experienced mandibular fracture and osteomyelitis after placement of multiple long implants in the mandible. Although the prevalence of mandibular fracture due to implant placement is very rare, reported at $0.2 \%$, nonunion of fracture, osteomyelitis, and osteonecrosis may occur and lead to serious problems unless timely diagnosis and treatment are not performed ${ }^{3)}$.

Fractures of the mandible can be diagnosed through radiographs along with clinical symptoms such as pain, redness, swelling, movement of bone fragments, and malocclusions in the affected area ${ }^{4)}$. When a mandibular fracture is diagnosed, the primary goal of fracture treatment is to stabilize the bone fragments during recovery ${ }^{5}$. And as in this case where osteomyelitis occurred due to delayed treatment of the fracture, in order to avoid further bone loss, active surgical treatment along with intensive antibiotic treatment should be performed. After the fractured segment is addressed, the stability should be secured through solid fixation, and proper surgical procedures such as curettage, saucerization, decortication, and sequestrectomy should be selected to appropriately treat osteomyelitis $^{7-9)}$. However in this case, the fracture site was re-fractured after the primary operation. The initial treatment approach that used only a single miniplate to 
fixate the bone was not sufficient due to the progression of osteomyelitis. Additionally for the case of secondary fracture, limitation of intermaxillary fixation due to the patient's edentulous condition and late detection of the patient's neurological symptoms contributed to the overall poor outcomes.

In order for an implant to be successful, it is very important to obtain excellent primary stability. Some researchers have advocated for the bicortical stabilization method which secures primary stability by placing an implant down to the lower cortical of the mandible when the residual bone mass is insufficient. However, multiple implant placements in the lower cortical disrupt the continuity of the lower cortical, which leads to a high risk of mandibular fracture ${ }^{10)}$. In this case, it is hypothesized that the fracture occurred due to a standard-length implant that was placed on the lower cortical bone of the mandible, despite mandibular atrophy. Therefore, for cases of severe alveolar bone atrophy in the mandibular posterior region, clinicians are recommended to use short implants ${ }^{11)}$. Short implant placement can prevent the need for surgical procedures such as alveolar bone reconstruction and minimize thermal damage and insertion torque due to excessive drilling which can prevent unnecessary invasion of anatomical structures such as the maxillary sinus and inferior alveolar nerve, and simplifies the procedure and allows for fewer bone grafts ${ }^{12}$. However, the use of wide implants to compensate for the short length of the implant can be a risk factor for mandibular fracture. It is important that at least $1 \mathrm{~mm}$ of cortical bone is retained on the buccal and lingual side of the implant after placement ${ }^{13)}$.

In this case, a re-fracture occurred due to an accidental fall during hospitalization. Falls are a serious problem in many hospitals and occur at a rate of $2.6 \sim 7 \%$; reports have indicated that $23 \sim 42 \%$ of falls lead to trauma to at least one site ${ }^{14)}$. In addition to physical harm such as fractures, a fall increases the treatment period and cost, lowers the patient's self-esteem, and can cause fear and anxiety ${ }^{15)}$. This patient might have been more susceptible to falls due to combinations of intrinsic factors such as advanced age, sex, and the side effects of medications and extrinsic factors such as general physical deterioration ${ }^{16)}$. In addition, there was a gap in medical staff attention and care for this case despite the patient's postoperative delirium symptoms. The symptoms and prevalence of postoperative delirium vary depending on the type and degree of surgery, and advanced age is a major risk factor ${ }^{17)}$. To prevent this, it is recommended that the degrees of anesthesia and sedation are minimized by using as much intraoperative monitoring as possible, that prolonged bed rest be avoided after surgery, and that the use of benzodiazepine drugs is minimized. A calendar and clock are recommended to facilitate the patient's orientation and should improve the quality of sleep ${ }^{18)}$. Considering the general risk factors for falls, special attention is required when managing hospitalization after surgery in elderly patients.

\section{CONCLUSION}

In elderly patients with severe bone atrophy, treatment with multiple implants should be selected with care. In such cases, clinicians should be aware of the high risk of mandibular fractures and therefore recommended to place short implants without compensating with wide implants. Despite the efforts, when mandible fractures accompanied by osteomyelitis occurs, it is crucial that timely reduction and active surgical treatment for osteomyelitis along with long-term antibiotic treatment is done in order to avoid further bone loss. Furthermore, for elderly patients, special attention is needed when hospitalizing considering the high possibility of secondary damage such as falls due to postoperative neurologic symptoms.

\section{CONFLICT OF INTEREST}

The authors have no financial relationships to disclose.

\section{ORCID}

Jun-Hyung Jeon, https://orcid.org/0000-0003-1691-5400 Jung-Gon Lee, https://orcid.org/0000-0002-1080-7401

Pil-Young Yun, https://orcid.org/0000-0001-6097-1229 Young-Kyun Kim, https://orcid.org/0000-0002-7268-3870 


\section{REFERENCES}

1. Raghoebar GM, Stellingsma K, Batenburg RH, Vissink A. Etiology and management of mandibular fractures associated with endosteal implants in the atrophic mandible. Oral Surg Oral Med Oral Pathol Oral Radiol Endod 2000;89:553-9.

2. Pieri F, Forlivesi C, Caselli E, Corinaldesi G. Short implants $(6 \mathrm{~mm})$ vs. vertical bone augmentation and standard-length implants ( $\geq 9 \mathrm{~mm}$ ) in atrophic posterior mandibles: a 5-year retrospective study. Int J Oral Maxillofac Surg 2017;46: 1607-14.

3. Kablan F, Abu-Sobeh A, Lorean A, Levin L. Spontaneous mandibular fracture as a complication of inferior alveolar nerve transposition and placement of endosseous dental implants in the atrophic mandible: a review of the literature and a report of two cases and their nonsurgical conservative management. Quintessence Int 2020;51:230-7.

4. Çağlayan F. TU. Cone beam computed tomography for accurate diagnosis of suspected fracture of the mandible. European Journal of Radiology Extra 2011;79.2:e51-e3.

5. Helfet DL, Haas NP, Schatzker J, Matter P, Moser R, Hanson B. AO philosophy and principles of fracture management-its evolution and evaluation. J Bone Joint Surg Am 2003;85: 1156-60.

6. Hogan A, Heppert VG, Suda AJ. Osteomyelitis. Arch Orthop Trauma Surg 2013;133:1183-96.

7. James R. Hupp EEI, Myron R. Tucker. Oral and Maxillofacial Surgery. 6th ed ed. St. Louis: Elsevier Inc., 2014.

8. Schmitz JP, Gassmann CJ, Bauer AM, Smith BR. Mandibular reconstruction in a patient with pyknodysostosis. J Oral Maxillofac Surg 1996;54:513-7.

9. Kato H, Matsuoka K, Kato N, Ohkubo T. Mandibular osteomye- litis and fracture successfully treated with vascularised iliac bone graft in a patient with pycnodysostosis. Br J Plast Surg 2005;58:263-6.

10. H.A. Emam HWF, C.A. Jatana. Management of atrophic mandible fractures: an updated comprehensive review. Oral Surgery 2018;11.1:79-87.

11. Spitzl C, Proschel P, Wichmann M, Heckmann S. Long-term neuromuscular status in overdenture and complete denture patients with severe mandibular atrophy. Int J Oral Maxillofac Implants 2012;27:155-61.

12. Kim SY, Ku JK, Kim HS, Yun PY, Kim YK. A retrospective clinical study of single short implants (less than $8 \mathrm{~mm}$ ) in posterior edentulous areas. J Adv Prosthodont 2018;10:191-6.

13. Oh WS, Roumanas ED, Beumer J, 3rd. Mandibular fracture in conjunction with bicortical penetration, using wide-diameter endosseous dental implants. J Prosthodont 2010;19:625-9.

14. Najafpour Z, Godarzi Z, Arab M, Yaseri M. Risk Factors for Falls in Hospital In-Patients: A Prospective Nested Case Control Study. Int J Health Policy Manag 2019;8:300-6.

15. Morello RT, Barker AL, Watts JJ, Haines T, Zavarsek SS, Hill KD, et al. The extra resource burden of in-hospital falls: a cost of falls study. Med J Aust 2015;203:367.

16. Stel VS, Smit JH, Pluijm SM, Lips P. Consequences of falling in older men and women and risk factors for health service use and functional decline. Age Ageing 2004;33:58-65.

17. Card E, Pandharipande P, Tomes C, Lee C, Wood J, Nelson D, et al. Emergence from general anaesthesia and evolution of delirium signs in the post-anaesthesia care unit. $\mathrm{Br} J$ Anaesth 2015;115:411-7.

18. Rengel KF, Pandharipande PP, Hughes CG. Postoperative delirium. Presse Med 2018;47:e53-e64. 